\title{
Queda no número de diagnósticos de cânceres durante pandemia de Covid-19: estadiamento e prognóstico prejudicados
}

\author{
Fall in the number of cancer diagnoses during the Covid-19 pandemic: impaired staging and \\ prognosis \\ Caída en el número de diagnósticos de cáncer durante la pandemia de Covid-19: deterioro de la \\ estadificación y el pronóstico
}

Bárbara Queiroz de Figueiredo ORCID: https://orcid.org/0000-0003-1630-4597 Centro Universitário de Patos de Minas, Brasil

E-mail: barbarafigueiredo@unipam.edu.br

Ana Caroline Barcelos Souza ORCID: https://orcid.org/0000-0003-2616-4874 Universidade de Uberaba, Brasil E-mail: anacarolinebarcelossouza@gmail.com

Brunna Gonçalves Machado ORCID: https://orcid.org/0000-0002-5510-5956 Centro Universitário de Patos de Minas, Brasil E-mail: brunnagm15@gmail.com

Cláudia Assis Siqueira ORCID: https://orcid.org/0000-0002-5138-5612

Centro Universitário Barão de Mauá, Brasil

E-mail: claudiaasiqueiraa@gmail.com

Gabriel Augusto Batista Alves

ORCID: https://orcid.org/0000-0002-9915-1129 Centro Universitário de Patos de Minas, Brasil

E-mail: gabrielaugusto84@ @otmail.com

João Pedro de Miranda Carvalho ORCID: https://orcid.org/0000-0001-8313-2903 Centro Universitário de Patos de Minas, Brasil E-mail: jp.mirandacv@gmail.com

Letícia Santos de Barros Moreira ORCID: https://orcid.org/0000-0002-2113-4126 Centro Universitário de Patos de Minas, Brasil

E-mail: leticiamoreira896@yahoo.com.br Luis Ferreira Neto ORCID: https://orcid.org/0000-0003-4397-2283 Universidade de Uberaba, Brasil

E-mail: trabalho.luis99@gmail.com Marianna Dutra Milagre ORCID: https://orcid.org/0000-0001-7800-9249 Universidade de Uberaba, Brasil

E-mail: mariannadutra.milagre@gmail.com Matheus Lemes Baliano

ORCID: https://orcid.org/0000-0002-5420-037X Universidade de Uberaba, Brasil

E-mail: matheusbaliano@gmail.com

\begin{abstract}
Resumo
Introdução: a atual pandemia de Covid-19 está tomando a saúde como refém. A redução do número de diagnóstico de câncer durante esse contexto, já evidenciada em muitos países, chama atenção para uma possível nova epidemia: a de casos de tumores avançados, em que certamente o prognóstico é pior, em um sistema de saúde, como o Sistema Único de Saúde (SUS), em que, sob condições normais, já apresenta dificuldade em oferecer tratamentos em prazos adequados. Objetivo: evidenciar o impacto que a Covid-19 causou nos pacientes oncológicos, ao mapeando procedimentos de rastreamento, diagnóstico e tratamento dos pacientes comparando com o período de 2019, sob cenário não pandêmico. Metodologia: trata-se de um estudo epidemiológico descritivo e quantitativo. Foi realizada a busca por incidência de cânceres no Brasil, durante os anos de 2019, 2020 e 2021, registrados no Sistema de Informação de Agravos de Notificação (SINAN) - DATASUS. Resultados e discussão: os motivos para este
\end{abstract}


fenômeno são variados, passando por recomendações dos próprios médicos em adiar consultas, cirurgias e exames de rotina, bem como redução da capacidade de atendimento de hospitais com vários profissionais de saúde doentes, mas, principalmente, o medo dos pacientes de saírem de casa para as consultas e exames, aliado ao receio de se contaminarem com o coronavírus nos hospitais ou clínicas ou até mesmo no transporte público. Conclusão: A redução do número de biópsias para diagnóstico de câncer, bem como de outros critérios diagnósticos, tem repercussão muito grande na mortalidade corroborada pelo câncer.

Palavras-chave: Covid-19; Câncer; Epidemiologia; Biópsia; Diagnóstico.

\begin{abstract}
Introduction: The current Covid-19 pandemic is taking health hostage. The reduction in the number of cancer diagnoses during this context, already evidenced in many countries, calls attention to a possible new epidemic: that of cases of advanced tumors, in which the prognosis is certainly worse, in a health system such as the System Health Service (SUS), in which, under normal conditions, it is already difficult to offer treatments within adequate terms. Objective: to show the impact that Covid-19 caused on cancer patients, by mapping procedures for tracking, diagnosis and treatment of patients compared to the period of 2019, under a non-pandemic scenario. Methodology: this is a descriptive and quantitative epidemiological study. The search for the incidence of cancers in Brazil was carried out during the years 2019, 2020 and 2021, registered in the Notifiable Diseases Information System (SINAN) - DATASUS. Results and discussion: the reasons for this phenomenon are varied, including recommendations from the doctors themselves to postpone appointments, surgeries and routine exams, as well as reducing the capacity of hospitals with several sick health professionals, but mainly fear of patients leaving home for consultations and exams, coupled with the fear of becoming contaminated with the coronavirus in hospitals or clinics or even on public transport. Conclusion: The reduction in the number of biopsies for the diagnosis of cancer, as well as in other diagnostic criteria, has a very large impact on mortality corroborated by cancer.
\end{abstract}

Keywords: Covid-19; Cancer; Epidemiology; Biopsy; Diagnosis.

\begin{abstract}
Resumen
Introducción: La actual pandemia de Covid-19 está tomando como rehenes a la salud. La reducción del número de diagnósticos de cáncer en este contexto, ya evidenciada en muchos países, llama la atención sobre una posible nueva epidemia: la de los casos de tumores avanzados, en los que el pronóstico es ciertamente peor, en un sistema de salud como el Sistema Salud. Servicio (SUS), en el que, en condiciones normales, ya es difícil ofrecer tratamientos en plazos adecuados. Objetivo: mostrar el impacto que causó el Covid-19 en los pacientes con cáncer, mediante el mapeo de los procedimientos de seguimiento, diagnóstico y tratamiento de los pacientes en comparación con el período de 2019, en un escenario no pandémico. Metodología: se trata de un estudio epidemiológico descriptivo y cuantitativo. La búsqueda de la incidencia de cánceres en Brasil se realizó durante los años 2019, 2020 y 2021 , registrados en el Sistema de Información de Enfermedades Notificables (SINAN) - DATASUS. Resultados y discusión: las razones de este fenómeno son variadas, incluyendo recomendaciones de los propios médicos para posponer citas, cirugías y exámenes de rutina, así como una reducción de la capacidad de los hospitales con varios profesionales de la salud enfermos, pero principalmente el miedo a que los pacientes se vayan de casa. para consultas y exámenes, sumado al miedo a contaminarse con el coronavirus en hospitales o clínicas o incluso en el transporte público. Conclusión: La reducción en el número de biopsias para el diagnóstico de cáncer, así como en otros criterios diagnósticos, tiene un impacto muy grande en la mortalidad corroborada por el cáncer.
\end{abstract}

Palabras clave: Covid-19; Cáncer; Epidemiología; Biopsia; Diagnóstico.

\title{
1. Introdução
}

O câncer é uma doença genética no sentido de que o fenótipo maligno resulta de uma alteração genética que é transmitida da célula alterada para suas células filhas. A cada divisão celular, o organismo humano sofre o efeito dos inúmeros agentes carcinógenos ambientais (Oliveira et al 2015; Silva et al. 2019). No entanto, o aparecimento e desenvolvimento de um clone de células tumorais é um evento relativamente raro. Isto ocorre porque a célula necessita romper uma série de barreiras fisiológicas para se tornar cancerígena. As barreiras mais primárias são os próprios pontos de controle do próprio ciclo celular (Mello et al. 2017).

A atual pandemia de Covid-19 está tomando a saúde como refém. Com um grande número de pacientes infectados globalmente e um número crescente de pacientes recém-diagnosticados, a doença representa um desafio sem precedentes para os sistemas de saúde (Silvestris et al. 2020). A crise desmantelou a forma como o atendimento é prestado e forçou os médicos a tomar difíceis decisões de triagem sobre quais tipos e componentes de atendimento têm valor imediato limitado e quais são essenciais para resultados ideais (Liang et al. 2020). No entanto, também é importante perceber que a mortalidade por outras 
doenças, como o câncer, permanece alta. Devido o cenário pandêmico, os profissionais de saúde enfrentam o desafio de reorganizar profundamente os sistemas de saúde em um ritmo sem precedentes, não apenas para lidar com a pandemia de Covid-19, mas também sem perder de vista o atendimento a outros pacientes. Como algumas doenças malignas podem representar uma ameaça imediata à sobrevivência, o câncer fornece uma lente para as principais mudanças em curso no atendimento clínico (Zhao et al. 2020.

A redução do número de diagnóstico de câncer durante esse contexto, já evidenciada em muitos países, chama atenção para uma possível nova epidemia: a de casos de tumores avançados, em que certamente o prognóstico é pior, em um sistema de saúde, como o Sistema Único de Saúde (SUS), em que, sob condições normais, já apresenta dificuldade em oferecer tratamentos em prazos adequados. Os motivos para este fenômeno são variados, passando por recomendações dos próprios médicos em adiar consultas, cirurgias e exames de rotina, bem como redução da capacidade de atendimento de hospitais com vários profissionais de saúde doentes, mas, principalmente, o medo dos pacientes de saírem de casa para as consultas e exames, aliado ao receio de se contaminarem com o coronavírus nos hospitais ou clínicas ou até mesmo no transporte público. (Onder et al. 2020). Dessa maneira, é possível que esses pacientes enfrentem muito mais dificuldades que o habitual pelo represamento desses diagnósticos, exames e cirurgias, que estão deixando de ocorrer. Por isso, o objetivo deste estudo é evidenciar o impacto que a Covid-19 causou nos pacientes oncológicos, ao mapeando procedimentos de rastreamento, diagnóstico e tratamento dos pacientes comparando com o período de 2019, sob cenário não pandêmico.

\section{Metodologia}

Trata-se de um estudo epidemiológico descritivo e quantitativo. Foi realizada a busca por incidência de cânceres no Brasil, durante os anos de 2019, 2020 e 2021, registrados no Sistema de Informação de Agravos de Notificação (SINAN) DATASUS, com base no recorte espacial dos meses referentes aos primeiros sintomas e diagnósticos. O presente trabalho foi realizado nas seguintes etapas: (I) pesquisa bibliográfica, (II) análise e coleta dos casos de casos diagnosticados de câncer nos anos de 2019, 2020 e 2021, (III) coleta de dados acerca dos principais procedimentos para o diagnóstico do câncer (IV) quantificação dos (V) análise dos casos de acordo com os meses de 2019, 2020 e 2021.

O presente estudo contou com o aporte de trabalhos acadêmicos entre os anos de 2018 e 2021. Por meio do acesso às principais bases de dados, a saber: dados National Library of Medicine (PubMed MEDLINE), Scientific Electronic Library Online (Scielo), Cochrane Database of Systematic Reviews (CDSR), Google Scholar, Biblioteca Virtual em Saúde (BVS) e EBSCO Information Services, no mês de agosto 2021. Para a compreensão visual dos resultados, utilizou-se dos dados disponibilizados pelo DATASUS para a construção de gráficos e tabelas. Por fim, a análise desses dados foi crucial para verificação de que a incidência de novos casos diagnosticados de cânceres diminuiu, de 2019 para 2020 e 2021.

\section{Resultados e Discussão}

A cada ano, no Brasil, cerca de 700 mil pessoas recebem o diagnóstico de câncer e 225 mil morrem por conta da doença. Em situações normais, o país já possui uma alta taxa de diagnóstico tardio, agora, com a pandemia, tudo indica que o problema vai aumentar muito (Carvalho et al. 2019). A Tabela 1 demonstra o número total de casos diagnosticados com câncer no ano de 2019, e a Tabela 2 ilustra o número de diagnósticos de cânceres no ano de 2020. 
Tabela 1: Número de diagnósticos de cânceres no ano de 2019.

\begin{tabular}{|c|c|}
\hline DIAGNÓSTICO & CASOS \\
\hline Neoplasias malignas & 361.614 \\
\hline Neoplasias in situ & 32.061 \\
\hline Neoplasias de comportamento incerto ou desconhecido & 71.250 \\
\hline Ignorado & 66.186 \\
\hline Total & 531.111 \\
\hline
\end{tabular}

Fonte: DATASUS: SIASUS (Sistema de Informações Ambulatoriais de Saúde - SUS) e SIHSUS (Sistema de Informações Hospitalares de Saúde - SUS)

Tabela 2: Número de diagnósticos de cânceres no ano de 2020.

\begin{tabular}{|c|c|}
\hline DIAGNÓSTICO & CASOS \\
\hline Neoplasias malignas & 327.710 \\
\hline Neoplasias in situ & 27.783 \\
\hline Neoplasias de comportamento incerto ou desconhecido & 70.444 \\
\hline Ignorado & 51.019 \\
\hline Total & 476.956 \\
\hline
\end{tabular}

Fonte: DATASUS: SIASUS (Sistema de Informações Ambulatoriais de Saúde - SUS) e SIHSUS (Sistema de Informações Hospitalares de Saúde - SUS).

Um dos principais procedimentos para o diagnóstico do câncer, as biópsias, tiveram uma redução de 39,11\%, quando comparados os meses de março a dezembro de 2019 e 2020. Em 2019, foram realizados 737.804 desses procedimentos e, em 2020, um total de 449.275. As maiores quedas ocorreram nos meses de abril (-63,3\%) e maio (-62,6\%).

Pelo Gráfico 1, nota-se que houve uma queda de aproximadamente 38,22\% no número total de biópsias, sendo o mês de abril de 2020 o que apresentou a maior queda. Outros exames importantes para a saúde da mulher, como o citopatológico cervico vaginal, tanto para diagnóstico, como para rastreamento de câncer do colo do útero, também apresentaram queda, assim como a mamografia. O Gráfico 2 expõe que 50,22\% foi a queda no número de exames citopatológicos com finalidade diagnóstica realizados no sistema público de saúde entre os meses de março a dezembro do ano de 2020 quando comparado com o ano de 2019, e o Gráfico 3 ilustra a queda no número de mamografias neste mesmo período, que alcançou $25,88 \%$ de redução. 
Gráfico 1: Número de biópsias realizadas no sistema público de saúde entre os meses de março a dezembro do ano de 2020 e de janeiro a abril de 2021, comparado ao ano de 2019.

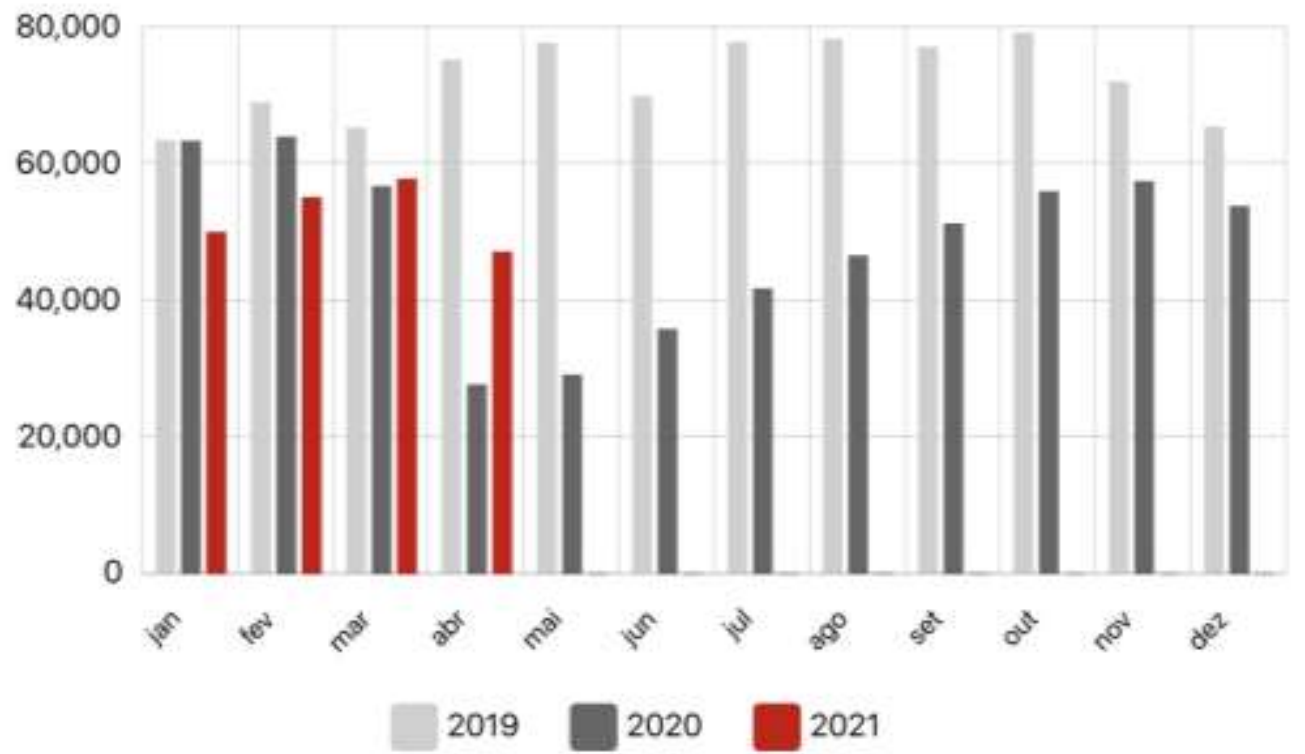

Fonte: DATASUS: SIASUS (Sistema de Informações Ambulatoriais de Saúde - SUS) e SIHSUS (Sistema de Informações Hospitalares de Saúde - SUS).

Gráfico 2: Número de exames citopatológicos com finalidade diagnóstica realizadas no sistema público de saúde entre os meses de março a dezembro do ano de 2020 e de janeiro a abril de 2021, comparado ao ano de 2019.

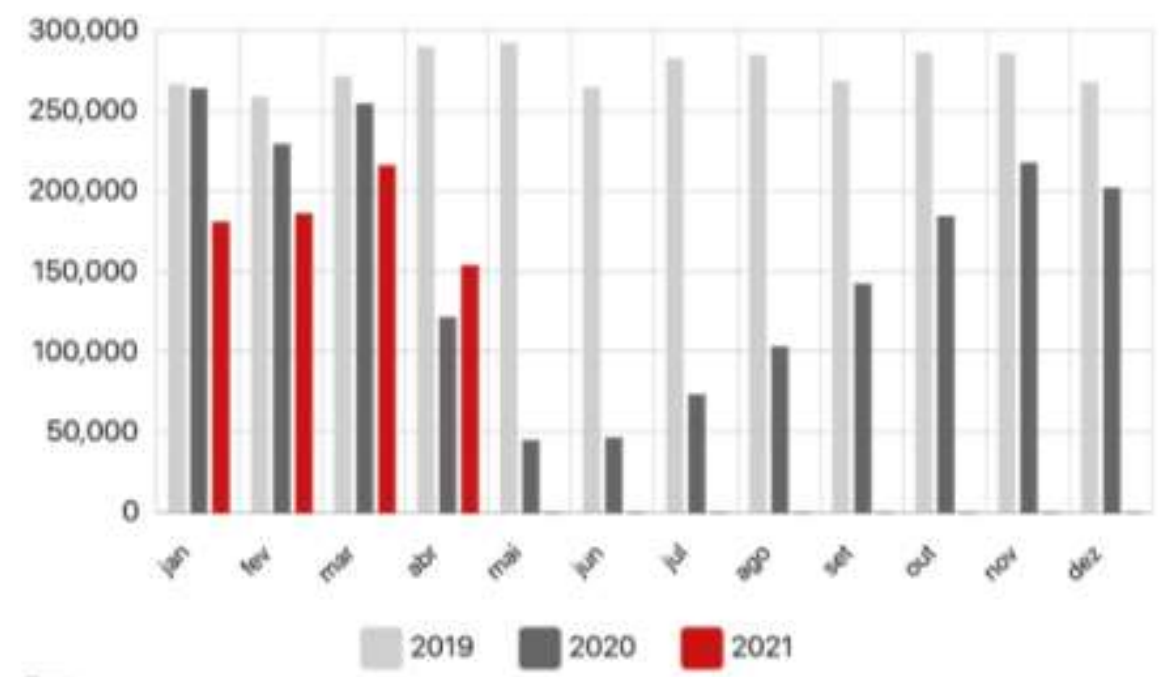

Fonte: DATASUS: SIASUS (Sistema de Informações Ambulatoriais de Saúde - SUS) e SIHSUS (Sistema de Informações Hospitalares de Saúde - SUS). 
Gráfico 3: Número de mamografias com finalidade diagnóstica realizadas no sistema público de saúde entre os meses de março a dezembro do ano de 2020 e de janeiro a abril de 2021, comparado ao ano de 2019.

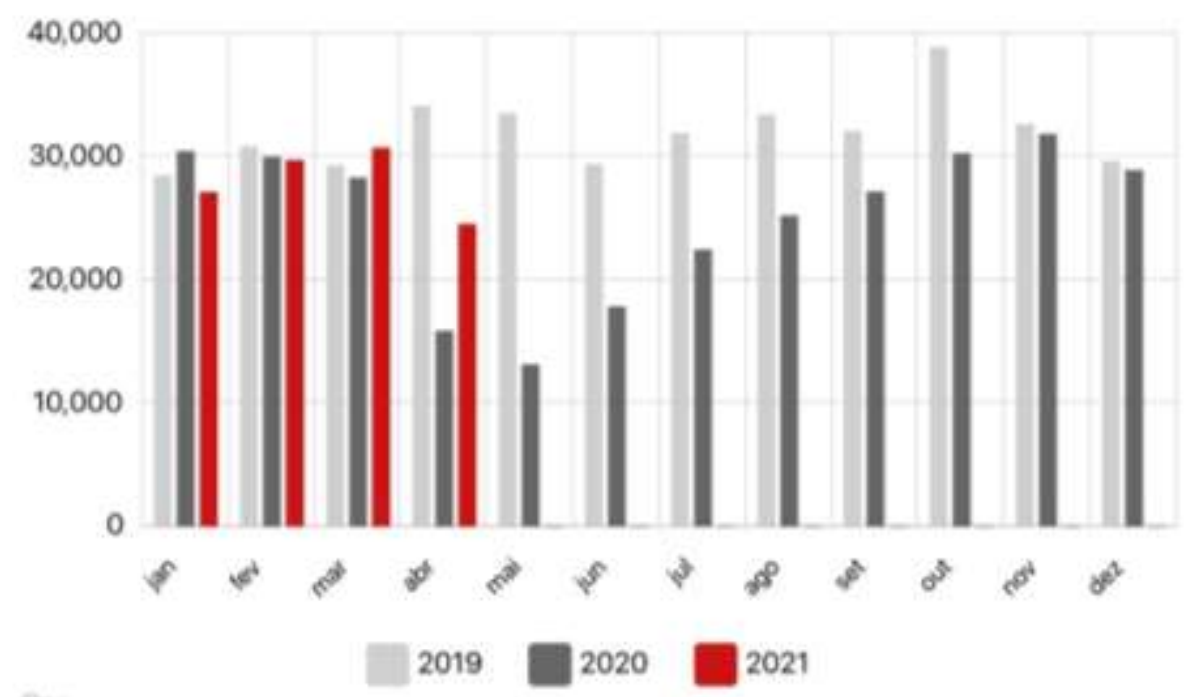

Fonte: DATASUS: SIASUS (Sistema de Informações Ambulatoriais de Saúde - SUS) e SIHSUS (Sistema de Informações Hospitalares de Saúde - SUS).

Sob essa perspectiva, $35,85 \%$ foi a queda no número de colonoscopias realizadas no sistema público de saúde entre os meses de março a dezembro do ano de 2020 quando comparado com o ano de 2019 e 29,04\% foi a queda no número de PSAs realizados no sistema público de saúde neste mesmo período.

Aliado a esses resultados, estudo de Bakouny et al. (2021) relatou uma diminuição significativa no número de pacientes submetidos a testes de rastreamento de câncer e no número de diagnósticos subsequentes de lesões cancerígenas e pré-cancerosas durante a pandemia de Covid-19 em um sistema de saúde no nordeste dos Estados Unidos. Verificou-se que, de junho a setembro de 2020, houve uma recuperação significativa no número de exames de rastreamento e diagnósticos subsequentes, a níveis quase pré-pandêmicos. Além disso, relatamos que o número de diagnósticos "perdidos" em potencial durante o período pandêmico primário foi provavelmente menor do que seria esperado porque a porcentagem de testes de triagem que levaram ao diagnóstico de uma lesão cancerosa ou pré-cancerosa foi maior durante o período pandêmico primário, o que pode refletir a priorização de pacientes de alto risco para rastreamento de câncer durante a pandemia (Marron et al. 2020).

O caos causado pela Covid-19 também trouxe mudanças substanciais no diagnóstico de câncer na Holanda. Dinmohamed et al. (2020) propõem que vários argumentos podem explicar essa diminuição. Em primeiro lugar, os indivíduos com sintomas potenciais não específicos de câncer podem ter barreiras para consultar um clínico geral, incluindo preocupações morais sobre a perda de tempo do clínico geral com sintomas não relacionados à Covid-19, suposições sobre capacidade insuficiente para sintomas essenciais. Serviços de saúde relacionados á infecção por SARS-CoV-2 e ansiedade sobre a aquisição da doença em um ambiente de saúde, bem como o fato de que a maioria das consultas de clínicos gerais para problemas não agudos serem transferidos para telessaúde.

Outrossim, hospitais podem ter adiado a avaliação diagnóstica ou ter tempos de resposta mais longos para a avaliação diagnóstica porque muitos recursos hospitalares estão sendo alocados para lidar com o Covid-19 acompanhamento (Schrag et al. 2020). Por último, os programas nacionais de rastreamento de câncer de mama, colorretal e colo do útero foram temporariamente suspensos a partir de 16 de março de 2020, na Holanda, a fim de aliviar a demanda no sistema de saúde devido ao Covid-19 (Amorim et al. 2020). Assim, o efeito dessa pausa no diagnóstico do câncer pode ser mais pronunciado 
após longos períodos de acompanhamento. No entanto, esse efeito pode ser menos notável para o câncer cervical porque o rastreamento visa identificar lesões pré-cancerosas (van de Haar et al. 2020). Coletivamente, menos diagnósticos de câncer na era Covid-19 resultarão de fatores do paciente, médico e do sistema. os programas nacionais de rastreamento de câncer de mama, colorretal e colo do útero foram temporariamente suspensos a partir de 16 de março de 2020, para aliviar a demanda no sistema de saúde devido a pandemia (Hanna et al. 2020).

\section{Conclusão}

O atendimento ao paciente oncológico pode ser interrompido devido à Covid-19 por meio de atrasos nas investigações diagnósticas e procedimentos cirúrgicos, bem como no diagnóstico de câncer atrasado devido à redução do rastreamento do câncer. $\mathrm{O}$ estudo demonstra, de fato, uma redução importante no acesso e na solicitação de exames, bem como no de diagnósticos realizados no período. A verificação de uma maior taxa de positividade nos exames durante o período de pandemia aponta para uma das possíveis estratégias para se reduzir o impacto da pandemia: o direcionamento equânime dos esforços e recursos para os pacientes com maior risco de desenvolver doenças, levando-se em conta fatores de risco de ordem biológica e social. São dados extremamente preocupantes, haja vista que a pandemia afetou e continua afetando profundamente o cenário do câncer: exames, tratamentos e consultas pré-agendados foram suspensos ou cancelados, tanto a pedido do paciente, como por medida de segurança adotada pelas instituições de saúde. A redução do número de biópsias para diagnóstico de câncer tem repercussão muito grande na mortalidade, pois, pela queda, muitas pessoas estão deixando de ser diagnosticadas e tratadas, o que permite que o tumor possa crescer e se tornar menos curável.

\section{Referências}

Amorim, G. L. S., Assad, D. X., Ferrari, B. L. \& Rosa, D. D. (2020). Breast oncology and the COVID-19 pandemic: Recommendations from the Brazilian Society of Clinical Oncology (SBOC). Brazilian Journal of Oncology, 16 (1), 1-8.

Bakouny, Z., Paciotti, M. \& Schmidt, A. L. (2021). Cancer screening tests and cancer diagnoses during the Covid-19 pandemic. JAMA Oncololy, 7 (3), 458 460.

Carvalho, J. B., \& Paes, N. A. (2019). Taxas de mortalidade por câncer corrigidas para os idosos dos estados do Nordeste brasileiro. Ciênc. saúde coletiva, 24(10), 3857-3866.

Dinmohamed, A. G., Visser, O., Verhoeven, R. H. \& Siesling, S. (2020). Fewer cancer diagnoses during the COVID-19 epidemic in Netherlands. The Lancet Oncology, 21 (6), 750-751.

Hanna, T. P., Evans, G. A. \& Booth, C. M. (2020). Câncer, COVID-19 e o princípio da precaução: priorizando o tratamento durante uma pandemia global. Nat Rev Clin., 17 (1), 268-270.

Liang, W., Guan, W. \& Chen, R. (2020). Pacientes com câncer na infecção por SARS-CoV-2: uma análise nacional na China. Lancet Oncology, 21 (3): 335 337

Marron, J. M., Joffe, S., Jagsi, R., Spence, R. A. \& Hlubocky, F. J. (2020). Ética e escassez de recursos: recomendações da ASCO para a comunidade oncológica durante a pandemia COVID19. J Clin Oncol., 38 (19), 1-5.

Mello, FA, Galle, LC, Prado, RL (2017). Prevenção do Câncer de Colo Uterino na Concepção da População Feminina de Uma Cidade do Interior do Estado de São Paulo. Colloquium Vitae, 9 (2): 45-52.

Oliveira, M. M., Malta, D. C., Guauche, H., Moura, L., \& Silva, G. A. (2015). Estimativa de pessoas com diagnóstico de câncer no Brasil: dados da Pesquisa Nacional de Saúde, 2013. Rev. Bras. Epidemiol, 18 (2), 146-157.

Onder, G., Rezza, G. \& Brusaferro, S. (2020). Taxa de letalidade e características de pacientes morrendo em relação ao COVID-19 na Itália. JAMA.

Santos, M. O. (2018). Estimativa 2018: Incidência de Câncer no Brasil. Rev Bras Cancerol, 64(1), 119-120.

Schrag, D., Hershman, D. L. \& Basch, E. (2020). Prática de oncologia durante a pandemia de COVID-19. JAMA.

Silva, F. S., Silva, G. S., Costa, A. C. M., Carvalho Filha, F. S. S., Medeiros Júnior, F. C., \& Câmara, J. T. (2019). Cuidados de enfermagem a pacientes oncológicos: revisão integrativa. Research., Society and Development, 8 (6), 1-9. 
Research, Society and Development, v. 10, n. 11, e273101119762, 2021

(CC BY 4.0) | ISSN 2525-3409 | DOI: http://dx.doi.org/10.33448/rsd-v10i11.19762

Silvestris, N., Moschetta, A., Paradiso, A., \& Delvino, A. (2020). Pandemia de COVID-19 e a crise dos sistemas de saúde: a experiência da Apulia Cancer Network e do Comprehensive Cancer Center Istituto Tumori "Giovanni Paolo II" de Bari. Jornal Internacional de Pesquisa Ambiental e Saúde Pública, 17 (8), 2763-2770.

van de Haar, J., Hoes, L. R. \& Coles, C. E. (2020). Cuidando de pacientes com câncer na era COVID-19. Nat Med., 26 (5), 665-671.

Zhao, N., Shi, J., Zeng, L., \& Yang, S. (2020). Características clínicas e estratégias de enfrentamento de neoplasias com infecção por coronavírus em 2019. Jornal Chinês de Câncer de Pulmão, 23 (4), 261-268. 